\title{
Ernährungsperiodisierung im Sport
}

\section{Kuno Hottenrott, Laura Hottenrott}

Sportler, die ihre Ernährung an das Training anpassen, können ihre Leistungsfähigkeit erhöhen und die Regeneration verbessern. Eine Ernährungsperiodisierung, wie das Intervallfasten oder der vorübergehende Verzicht auf eine energetisch ausreichende Nahrungsaufnahme, kann die Reizwirksamkeit des Trainings erhöhen. Davon profitieren insbesondere Sportler, die eine Veränderung der Körperzusammensetzung bewirken wollen.

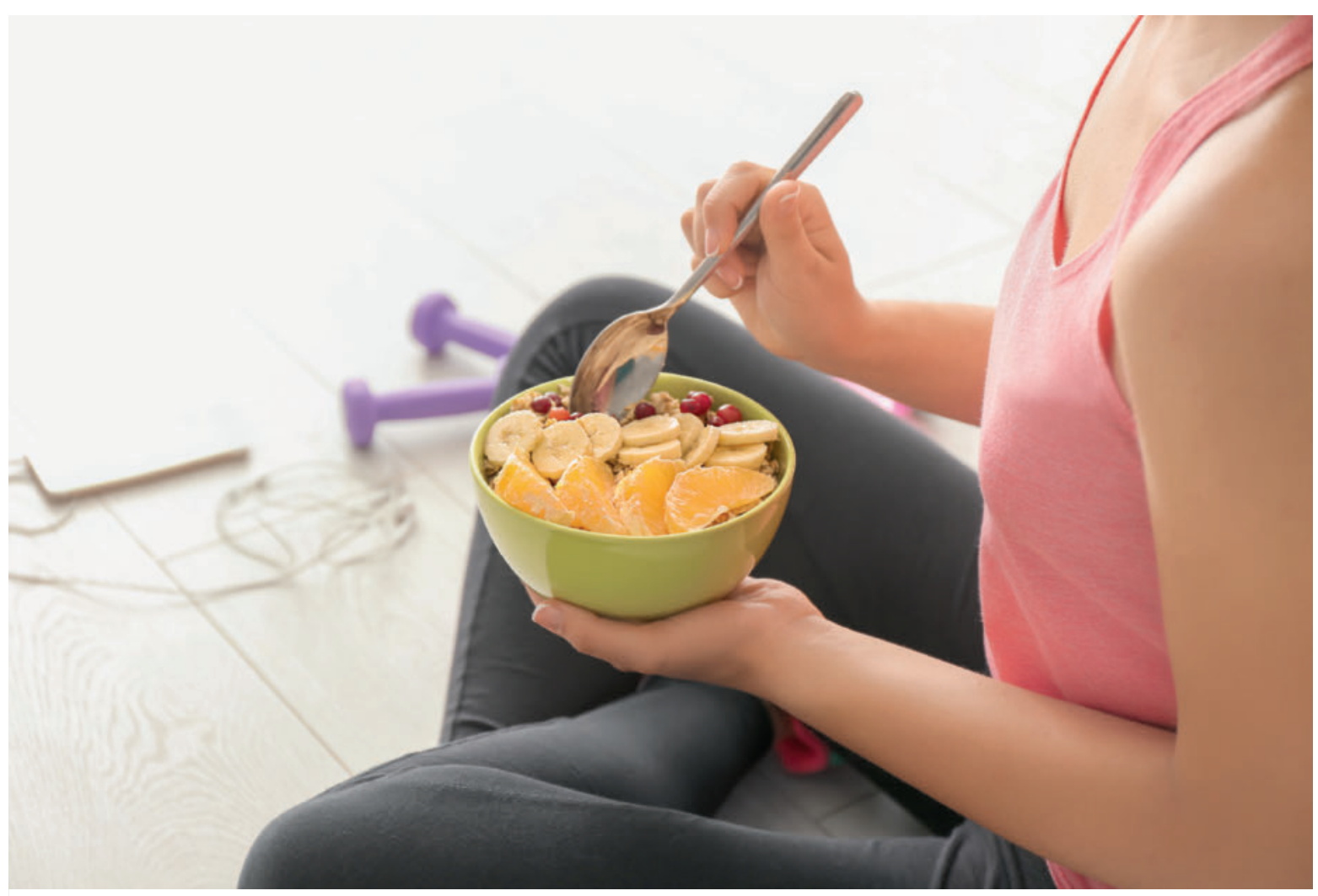

- Abb. 1 Eine Periodisierung der Ernährung kann dazu beitragen, die Leistungsfähigkeit zu erhöhen. Foto: Pixel-Shot/Adobe Stock

Die Ernährung ist eine wichtige Säule sportlicher Leistungen und hat großen Einfluss auf Regeneration und Gesundheit. Eine basische, ausgewogene und natürliche Ernährung bildet dazu die Grundlage. Ein ausgeglichener Säure-Basen-Haushalt ist Voraussetzung für zahlreiche Stoffwechselprozesse im Organismus. Bestimmte Lebensmittel bilden bei der Verstoffwechselung Säuren, andere Basen. Tierische Proteine in Fleisch, Wurst und Milchprodukten sowie Getreide, einige Hülsenfrüchte und Softdrinks gehören zu den säurebildenden Lebensmitteln. Obst und Gemüse werden aufgrund einer hohen Dichte an Mineralien den Basenbildnern zugeschrieben. Eine ernährungsbedingte Übersäuerung (latente Azidose), ausgelöst durch eine basenarme Ernährung, begünstigt Entzündungen, kann eine Vielzahl von Erkrankungen und Beschwerden hervorrufen sowie die Leistungsfähigkeit vermindern. Das Binde- und Stützgewebe dient als Speicherort für Säuren und verliert bei einer latenten Azi- 
dose unter anderem an Elastizität, was beispielsweise Probleme an Sehnen hervorrufen und Trainingsunterbrechungen zur Folge haben kann.

Neben einer basischen Ernährungsweise haben die Makronährstoffe beim Sport eine besondere Bedeutung. Eine ausgewogene und gezielte Aufnahme von Kohlenhydraten, Fetten und Proteinen sichert die Leistungsfähigkeit und kann die muskuläre Regeneration beschleunigen. Die Makronährstoffe müssen zudem stets an die Anforderungen des sportlichen Trainings in der Sportart hinsichtlich Auswahl, Verteilung und Periodisierung angepasst werden ( $\triangleright$ Abb. 1).

\section{Proteine}

Der Mensch kann Proteine nur in geringen Mengen speichern. Die tägliche Aufnahme von 0,8g Protein pro kg Körpergewicht wird allgemein empfohlen, sie ist aber für Leistungssportler zu niedrig. Bei hohen Trainingsumfängen (>8Stunden/Woche) oder wenn Muskelmasse durch Krafttraining aufgebaut werden soll, können 1,6-2,4g Protein pro kg Körpergewicht aufgenommen werden. [1]. Der erhöhte Bedarf sollte dabei vorwiegend über pflanzliches Protein gedeckt werden. Durch eine günstige Kombination verschiedener Lebensmittel (z. B. Kartoffeln mit Ei) kann die biologische Wertigkeit der Eiweiße erhöht werden.

\section{Fette}

Fette haben viele wichtige Funktionen im Körper. Um die Gefäße zu schützen und die Nervenzellen optimal zu versorgen, benötigen wir nicht nur einen hohen Gehalt an mehrfach ungesättigten Fettsäuren in unserer Nahrung, sondern auch ein günstiges Verhältnis zwischen den Omega-6- und Omega-3-Fettsäuren. Letztere werden oft zu wenig konsumiert. Die mehrfach ungesättigten Omega-3-Fettsäuren EPA und DHA verhindern die Entstehung von Entzündungen, während Omega-6-Fettsäuren Entzündungsprozesse fördern können [14]. Bei Sportverletzungen und Muskelschmerzen können durch eine erhöhte Aufnahme von Omega-3-Fettsäuren Muskelkater reduziert und Entzündungsparameter gesenkt werden [15]. Für Sportler werden nach allgemeinen Richtlinien EPA und DHA von etwa 1-2 g/d bei einem Verhältnis von EPA:DHA von 2:1 gegeben [16]. Nutzbare Quellen für Omega-3Fettsäuren sind Leinsamenöl, Walnussöl, Rapsöl, Fischöle, Kaltwasserfisch sowie Nüsse. Insgesamt sollte die Ernährung möglichst natürlich und vollwertig ausgerichtet sein sowie wenig industriell verarbeitete Produkte enthalten. Viele Fertigprodukte enthalten oft wenig Vitamine und Mineralien, aber meist Konservierungsstoffe sowie andere Zusatzstoffe. Dies sind Farbstoffe, Geschmacksverstärker und vor allem auch viel (Frucht-)Zucker, Sirup sowie Transfettsäuren.

Empfehlungen zur allgemeinen Sporternährung müssen auch das Athletenalter und Geschlecht berücksichtigen. Spezielle Anforderungen gelten für Heranwachsende, Al- tersklassenathleten und für Frauen [2]. So beeinflusst der monatliche Zyklus der Frau die Leistungsfähigkeit, den Energieumsatz und den Eisenstoffwechsel [3]. Die im Folgenden beschriebenen Strategien zur Ernährungsperiodisierung beziehen sich auf Athleten im Erwachsenenalter. Ziel des Artikels ist es, Strategien darzulegen, die dazu beitragen, die Leistungsfähigkeit des Breiten- und Leistungssportlers durch eine auf das Training abgestimmte Ernährungsperiodisierung zu steigern.

\section{Ernährungsperiodisierung}

Ernährungsperiodisierung beschreibt die systematische Planung der Nahrungsaufnahme in Abstimmung mit den Inhalten des sportlichen Trainings mit dem Ziel, Anpassungen an das Training zu optimieren, die Regeneration zu beschleunigen und die Leistungsfähigkeit zu erhöhen.

\section{Periodisierung der Mahlzeitenhäufigkeit}

Im Leistungssport, insbesondere in den Ausdauersportarten mit hohem Trainingspensum, sind in der Regel 3 Hauptmahlzeiten zu festgelegten Zeiten nicht realisierbar. Die Nahrungsaufnahme wird stattdessen den Trainingszeiten angepasst. Kleinere Zwischenmahlzeiten kön- 


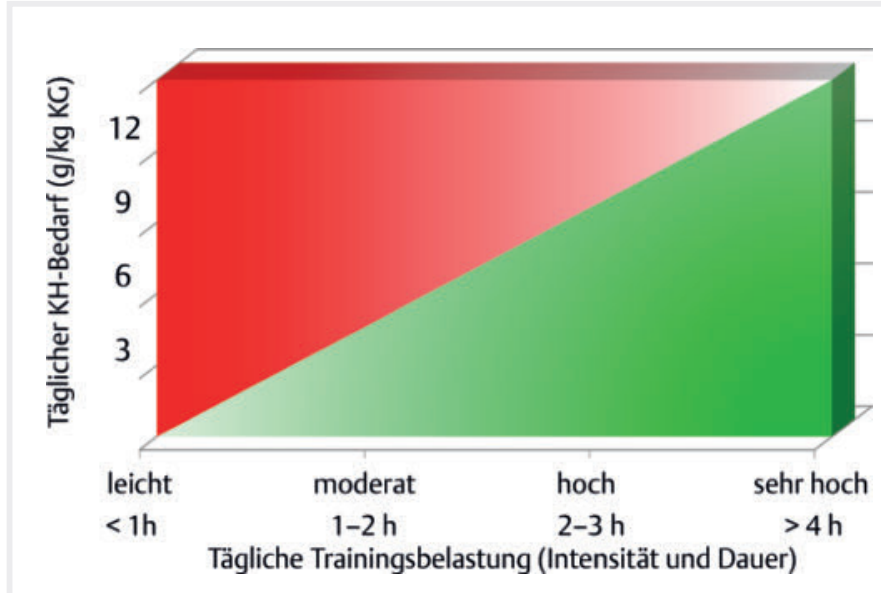

Abb. 2 Kohlenhydratbedarf in Abhängigkeit der Trainingsbelastung [8].

nen die Leistungsfähigkeit halten und die Regeneration beschleunigen. Durch einen bewussten Verzicht auf Nahrungsaufnahme wie beim Intervallfasten, kann die Wirksamkeit des Trainings erhöht, gesundheitsrelevante Faktoren können begünstigt und Entzündungsprozessen kann entgegengewirkt werden. Die Mechanismen der Unterbrechung der Nahrungsaufnahme führen zu einer Umstellung auf den Fettstoffwechsel, einer erhöhten Ketonkörperproduktion und einer Stimulation der adaptiv-zellulären Stressreaktionen [4].

Merke

Beim Leistungssport wird die Nahrungsaufnahme den Trainingszeiten angepasst.

\section{Periodisierung der Gesamtenergieaufnahme}

Beim Sport variiert die Gesamtenergieaufnahme beachtlich, insbesondere in Abhängigkeit von der Höhe der Trainingsbelastung. Ein systematischer Trainingsaufbau weist zyklisch wiederkehrende Belastungs- und Entlastungsphasen auf, die akribisch geplant werden. Die Energieaufnahme muss entsprechend fortwährend dem trainingsbedingten Mehr- oder Minderbedarf angepasst werden. Ziel der Energieaufnahme von ambitionierten Sportlern sollte es sein, eine ausgeglichene tägliche Energiebilanz zu erreichen, um die Leistungsfähigkeit aufrechtzuerhalten.

Jedoch gibt es auch Phasen, in der die Energiebilanz positiv oder negativ ausgerichtet werden muss, um Körpergewicht oder Körperfett zu reduzieren oder Muskelund Körpermasse zu erhöhen, wie in den Körpergewichtsklassen der Kampfsportarten, im Gewichtheben oder im Rudern. Um das Wettkampfgewicht zu erzielen, werden teilweise drastische Maßnahmen, die unter dem Begriff Gewichtmachen oder Abkochen im Sport bekannt sind, durchgeführt. Um Leistungsverlust, Infektanfälligkeit und Trainingsqualitätseinbußen in solchen Phasen zu minimieren und um eine hohe Wettkampfleistung zu erzielen, muss eine strategische Periodisierung der Energieaufnahme erfolgen.

\section{Periodisierung der Makronährstoffe}

Als optimale Makronährstoffverteilung für Erwachsene werden $55 \%$ Kohlenhydrate, $30 \%$ Fett und $15 \%$ Protein empfohlen [5]. Im Sport ist diese Verteilung nicht immer sinnvoll und sollte stattdessen den Trainingszielen fortwährend angepasst werden. So wird beispielsweise im Rahmen eines Kraftzyklus zur Steigerung der Muskelleistung eine erhöhte Proteinaufnahme und im Rahmen einer allgemeinen Vorbereitungsperiode während eines langen aeroben Ausdauertrainings oder einer langen Wanderung zur Induktion der Energiegewinnung aus Fetten eine geringere Kohlenhydrataufnahme empfohlen. Die Empfehlungen zur Verteilung der Makronährstoffe sind auch in den Sportartengruppen unterschiedlich und variieren zusätzlich in Abhängigkeit der Trainingsperiode. Während in der allgemeinen Vorbereitungsperiode der Proteinanteil höher ist, nimmt in der Wettkampfperiode der Kohlenhydratanteil zu [6]. In einer randomisiert placebokontrollierten Studie [7] ließ sich zeigen, dass eine Aufnahme von 30-40 g Casein-Protein 30 Minuten vor dem Schlafengehen zur Verbesserung der Regeneration und zur Steigerung der Muskelproteinsynthese über Nacht führt. Mit einer gezielten Periodisierung der Makronährstoffe im Trainingsprozess kann so die Wirksamkeit des Trainings erhöht werden.

\section{Strategien der Ernährungs- und Trainingsperiodisierung}

\section{Kohlenhydrataufnahme in Training und Wettkampf}

Ausdauertraining wird in unterschiedlichen Belastungszonen (Intensitätsbereichen) durchgeführt. Während das niedrig intensive Grundlagenausdauertraining (GA1) bei geringer Kohlenhydratverfügbarkeit auf die Verbesserung des Fettstoffwechsels zielt, nehmen die Kohlenhydrate bei höheren Belastungsintensitäten eine bedeutende Rolle ein. Welche Substrate beim Sport vorrangig genutzt werden, wird nicht nur von der gewählten Trainingsintensität bestimmt, sondern auch von der Verfügbarkeit der Kohlenhydrate und dem Speicherstatus des Glykogens im Muskel und in der Leber. Bei intensiven Ausdauerbelastungen von über einer Stunde Dauer führt die Aufnahme von Kohlenhydraten in Form von Energiedrinks, -riegeln oder -gelen alle $20 \mathrm{~min}(80 \mathrm{~g} / \mathrm{h}$ ) zu einer signifikanten Leistungssteigerung. In Anlehnung an Burke et al. [8] werden je nach Trainingsintensität und Trainingsvolumen folgende Empfehlungen für die Kohlenhydrataufnahme gegeben ( $\triangleright$ Abb. 2).

\section{Low carb - train low}

Mit der Strategie „low carb - train low“ soll eine erhöhte Reizsetzung auf die Fettstoffwechselaktivität und Mitochondrienbiogenese erzielt werden ( $\triangleright$ Abb. 3). Bei nied- 
riger Kohlenhydratverfügbarkeit werden längere Ausdauertrainingseinheiten in niedriger Intensität (high volume training) absolviert. Der Organismus wird in seiner Fähigkeit trainiert, mehr Energie aus Fettsäuren bereitzustellen. Für die Umsetzung dieses Konzeptes gibt es verschiedene Möglichkeiten:

a. Die Glykogenspeicher werden am Abend nach dem Training nicht durch eine vermehrte Kohlenhydrataufnahme (<3g/kg Körpergewicht) wieder aufgefüllt. Stattdessen wird auf eine weitere Entleerung über Nacht (sleep low, overnight fasting) gesetzt [9].

b. Nach einer intensiven Trainingseinheit werden die entleerten Glykogenspeicher nicht wieder aufgefüllt (recovery low) [6][9].

c. Das Ausdauertraining beginnt erst nach einer mindestens 16-stündigen Intervallfastenphase (train fasted) [11].

\section{High carb - train high}

Hohe Trainingsintensitäten unter Nutzung schneller Muskelfasern setzen die Verfügbarkeit schneller Energiequellen voraus. Die „high carb - train high“-Strategie kann durch eine gezielte (mehrtägige) Auffüllung der zuvor entleerten muskulären Glykogenspeicher (Superkompensation) und durch eine zusätzliche Aufnahme von Kohlenhydraten unmittelbar vor und während der sportlichen Aktivität bzw. des Wettkampfes (race nutrition) umgesetzt werden [9]. Auch das hochintensive Intervalltraining (HIIT) profitiert von der Strategie schnell verfügbarer Energie.

\section{Low carb - high fat}

Ziel einer „low carb - high fat“ (LCHF) bzw. ketogenen Diät ist es, Vorteile durch eine längerfristige (mehrtägige bis mehrwöchige) Anpassung an die Fettoxidation mit einhergehend hohen Konzentrationen an zirkulierenden Ketonkörpern zu erreichen. Bei der ketogenen Diät besteht die Ernährung hauptsächlich aus Fetten und Proteinen. Die Kohlenhydratzufuhr wird drastisch auf etwa $5 \%$ der täglichen Energiezufuhr minimiert. Das Verarmen des Organismus an Kohlenhydraten als Energieträger bedingt nach einer Anpassungszeit von etwa 3 Tagen ein Umstellen auf den ketogenen Stoffwechsel [4]. Der Zustand dieses Energiestoffwechsels zeichnet sich durch einen vermehrten Abbau von freien Fettsäuren aus, welche aufgrund des Kohlenhydratmangels zu Ketonkörpern (Betahydroxybutyrat, Acetacetat und Aceton) umgebaut werden. Diese Produkte der Betaoxidation der Fettsäuren liefern im Hungerzustand etwa 5-7\% der Energie, besonders für den Hirnstoffwechsel. Essenziell ist hierbei, dass der Fettstoffwechsel optimiert und die Ketonkörper als Ersatzenergiequelle (Ersatzkohlenhydrat) genutzt werden. Der Organismus mobilisiert Fette, wodurch auch eine Reduktion der Körperfettmasse erzielt werden kann.

In Studien konnte bei Ausdauerathleten nach mehrwöchiger LCHF-Diät eine erhöhte Fettoxidationsrate bei niedri-

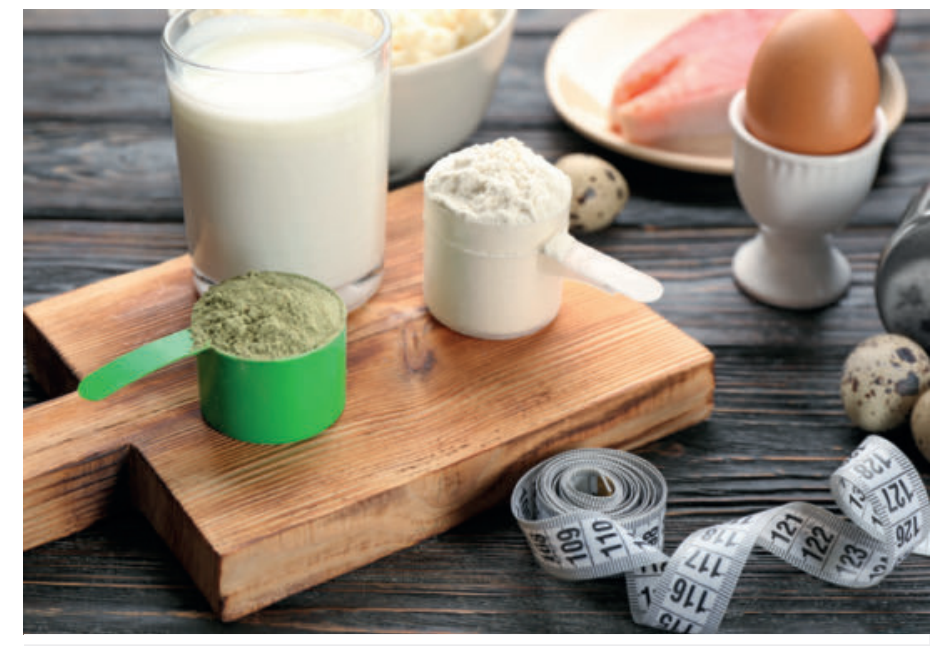

Abb. 3 Low-carb- und High-carb-Phasen können im Training strategisch eingesetzt werden. Foto: Africa Studio/Adobe Stock

gen und moderaten Belastungen festgestellt werden. Jedoch beeinträchtigte die LCHF-Diät die Fähigkeit, in hohen Intensitätsbereichen zu trainieren, sodass maximale Leistungen nicht erzielt werden konnten [6]. Insofern ist eine mehrtägige LCHF-Diät nur für die allgemeine Vorbereitungsperiode und nicht für die Wettkampfphase zu empfehlen. Eine Ausnahme stellt die sogenannte Saltin-Diät [12] in der unmittelbaren Vorbereitung beispielweise auf einen Marathon oder Triathlon dar. Nach einer 3-4-tägigen „low carb - high fat“-Phase folgen 3-4 High-carb-Tage bis zum Wettkampf, um eine erhöhte Füllung der Muskel- und Leberglykogenspeicher zu erzielen.

\section{KERNAUSSAGEN}

Zusammenfassend zeigen die obigen Ausführungen, wie eine durchdachte Ernährungsweise zur Optimierung der Leistung im Sport beitragen kann. Die Reizwirksamkeit des Trainings kann durch eine Ernährungsperiodisierung ganzjährig erhöht werden. Von der Maßnahme des Intervallfastens profitieren nicht nur Leistungssportler, sondern auch Breitensportler, vor allem dann, wenn neben der sportlichen Belastung eine Veränderung der Körperzusammensetzung in Verbindung mit einer Gewichtsreduktion angestrebt wird. Die Strategie „low carb - train low“ in Kombination mit Intervallfasten und einer zusätzlichen Supplementierung von basischen Mineralien, als Schutz vor einer Übersäuerung des Körpers, tragen zur Verbesserung des Fettstoffwechsels und einer verstärkten Gewichtsabnahme bei [13]. Der intermittierende Verzicht auf energetisch ausreichende Nahrungsaufnahme hat zudem weitere positive gesundheitliche Wirkungen unter anderem auf erhöhte Blutdruck- und Blutfettwerte, Insulinsensibilität, Entzündungsparameter, oxidativen Stress [4]. 


\section{Interessenkonflikt}

Die Autoren erklären, dass keine wirtschaftlichen oder persönlichen Verbindungen bestehen.

\section{Erstveröffentlichung}

Dieser Beitrag ist zuerst erschienen in zkm - Zeitschrift für Komplementärmedizin 2020; (1): 34-37.

Autor

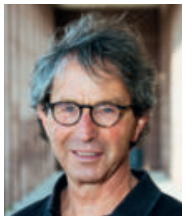

Kuno Hottenrott

lehrt und forscht seit 2003 am Institut fü Sportwissenschaft der Martin-Luther-Universität Halle, ist Leiter des Arbeitsbereichs Trainingswissenschaft \& Sportmedizin und Direktor des Instituts für Leistungsdiagnostik und Gesundheitsförderung (ILUG). Hottenrott hat über 20 Monografien und über 150 Fachartikel zu den Themen Leistungs- und Gesundheitssport, Sporternährung, Leistungsdiagnostik sowie zur autonomen und zentralnervalen Kontrolle lokomotorischer Bewegungen im Sport veröffentlicht und ist Pastpräsident der Deutschen Vereinigung für Sportwissenschaft (dvs). Seine Bestzeit im Marathon ist 2:36h.

Korrespondenzadresse

Univ.-Prof. Dr. Kuno Hottenrott

Institut für Sportwissenschaft

Martin-Luther-Universität Halle-Wittenbers

Von-Seckendorff-Platz 2

06120 Halle (Saale)

kuno.hottenrott@sport.uni-halle.de
[2] Neumann G. Ernährung im Sport. 8. Aufl. Aachen: Meyer \& Meyer; 2016

[3] Desbrow B, Burd NA, Tarnopolsky M et al. Nutrition for special populations: young, female, and masters athletes. Int I Sport Nutr Exerc Metab 2019; 29: 220-227

[4] Longo VD, Mattson MP. Fasting: molecular mechanisms and clinical applications. Cell Metab 2014; 9: 181-192

[5] DACH - Deutsche Gesellschaft für Ernährung, Österreichische Gesellschaft für Ernährung, Schweizerische Gesellschaft für Ernährung, Hrsg. Referenzwerte für die Nährstoffzufuhr. Bonn: Neuer Umschau Buchverlag; 2018

[6] Jeukendrup AE. Periodized nutrition for athletes. Sports Med 2017; 47: 51-63

[7] Res P, Groen B, Pennings B et al. Protein ingestion before sleep improves postexercise overnight recovery. Med Sci Sports Exerc 2012; 44: 1560-1569

[8] Burke LM, Hawley JA, Wong SH et al. Carbohydrates for training and competition. J Sports Sci 2011; 29: 17-27

[9] Marquet LA, Hausswirth C, Molle $O$ et al. Periodization of carbohydrate intake: Short-term effect on performance. Nutrients 2014; 8: 755

[10] Stellingwerff T, Morton JP, Burke LM. A framework for periodized nutrition for athletics. Int J Sport Nutr Exerc Metab 2019; 29: 141-151

[11] Hottenrott K, Meyer T, Huesmann J et al. Das LIF-Konzept. Laufen und Intervallfasten für Figur und Fitness. Wenn Wissenschaft in der Praxis überzeugt. Eigenverlag; 2016

[12] Karlsson J, Saltin B. Diet, muscle glycogen, and endurance performance. J Appl Physiol 1971; 31: 203-206

[13] Hottenrott K, Hottenrott L. Intermittierendes Fasten und Sport. Schweiz Z Ganzheitsmed 2017; 29: 265-268

[14] von Schacky C. Omega-3 index and cardiovascular health. Nutrients 2014; 6: 799-814

[15] Kim J, Lee J. A review of nutritional intervention on delayed onset muscle soreness. Part I. J Exerc Rehabil 2014; 10: 349-356

[16] Simopoulos AP. Omega-3 fatty acids and athletics. Curr Sports Med Rep 2007; 6: 230-236

Bibliografie

DOI https://doi.org/10.1055/a-1113-2877

Ernährung \& Medizin 2020; 35: 32-36

(c) Georg Thieme Verlag KG Stuttgart · New York ISSN 1439-1635 